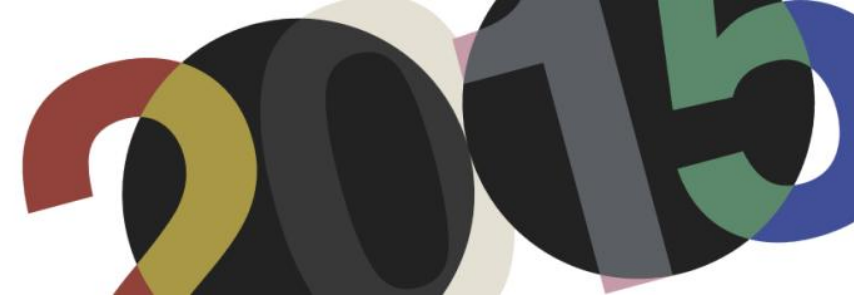

DOI: http://dx.doi.org/10.4995/LC2015.2015.764

\title{
Analyse architecturale, modélisation 3D et narration filmique : un regard original sur quelques objets corbuséens
}

\author{
D. Derycke*, V. Boone ** \\ * ULB, Bruxelles, Faculté d'architecture, CLARA/AlICe \\ ** ULB, Bruxelles, Faculté d'architecture, CLARA/hortence; ENSAP Lille, LACTH
}

\begin{abstract}
To analyze Le Corbusier's work through 3D digital modeling constitutes an important issue for the dissemination of the legacy of this major architect. During an analytical process, a relevant use of contemporary graphic means associated to an understanding of the codes of architectural representation allows to reveal new issues, or new points of view. When linking the graphic production of such process to film narrative and to the codes of documentary film, one obtain singular documents: short-movies based on computer generated images that support an analytical and critical thought, but also present projects under a new visual expression; didactic and descriptive. Those documents become particularly interesting when it comes to highlighting obscure architectural heritage, or to give body to projects remained on paper. With the support of the Fondation Le Corbusier, some Master architecture students investigated Belgian projects of the work of Le Corbusier, from which only two were built and no more than one remains. Through precise methodological issues, this paper accounts for the knowledge that such productions can offer on sometimes less-known architectural objects from the Swiss master.
\end{abstract}

Resumen: Analizar las obras arquitectónicas de Le Corbusier, a través la modelización 3D, constituye un desafío importante de difusión del legado de este gran arquitecto. Mediante el proceso analítico, un uso pertinente de los medios contemporáneos de representación gráfica asociados a una comprensión de los códigos de representación arquitecturales permite de establecer nuevas perspectivas y problemáticas poco tratadas. Cuando acoplamos la producción gráfica, creada por este tipo de proceso analítico, a la narración cinematográfica y a los códigos del cine documental, obtenemos documentos singulares : cortometrajes dentro de imágenes digitales que sostienen un propósito analítico y critico, pero que su vez presentan también los proyectos arquitectónicos bajo una nueva expresión visual, didáctica y descriptiva. Estos documentos pueden revelarse particularmente interesantes cuando se trata de valorizar un patrimonio arquitectural desconocido, o de ofrecer un cuerpo solido a los proyectos yacidos en hojas de papel. Con el apoyo de la Fundación Le Corbusier, los estudiantes de Master de arquitectura se interesaron a los proyectos belgas de los trabajos diseñados por Le Corbusier, de los cuales solo dos han sido construidos y solo uno permanece en pie. Mediante cuestionamientos metodológicos precisos, este articulo relata el conocimiento que este tipo de producciones pueden ofrecer sobre los objetos arquitectónicos menos conocidos del gran maestro suizo.

Keywords: Le Corbusier, architectural analysis, 3D modelisation, photography, short film, Belgium.

Palabras clave: Le Corbusier, análisis arquitectónico, modelización 3D, fotografía, cortometrajes, Bélgica.

\section{Introduction}

Le laboratoire AlICe de la Faculté d'Architecture de l'ULB s'intéresse depuis plus de 20 ans à l'analyse architecturale par la représentation graphique. Un des exercices proposé à des étudiants de Master consiste à analyser et à modéliser en 3D un objet d'architecture, puis à traduire cette analyse en un court-métrage en images de synthèse, en tenant un discours à la fois didactique et critique sur le bâtiment étudié. Ce travail permet aux étudiants de développer leur capacité à lire l'architecture d'une part, et d'autre part d'apprendre à construire, structurer et communiquer un propos précis à travers un média particulier (images de synthèse enrichies de prises de vues filmées, images d'archives, musiques, voix off,...). Cette approche pédagogique permet de mêler 
l'histoire et la théorie de l'architecture à la compréhension de la représentation graphique et de ses codes, anciens et nouveaux, avec comme objectif final un possibilité de diffusion du savoir architectural par les réseaux médiatiques contemporains. Souvent prétexte à des partenariats avec des institutions extérieures en charge de la gestion de patrimoine architectural, cet exercice prend une tournure particulièrement intéressante lorsqu'il s'agit de valoriser des fonds d'archives, des projets non-construits, ou encore des bâtiments disparus ou fortement altérés. Dans le cas de projets non-réalisés ou disparus, la maquette 3D permet de dépasser la simple question volumétrique contenue dans les documents originaux ou subsistants, et d'introduire pleinement la question de la spatialité, souvent cantonnée à quelques croquis ou photographies de maquettes. En outre, des informations parfois contradictoires provenant des documents sources doivent faire l'objet d'interprétations justifiées afin de cohabiter dans un même espace de représentation. Il résulte de ce processus des objets graphiques singuliers, qui soulèvent non seulement des enjeux de recherches, mais qui ont également un impact dans le domaine didactique et de la communication.

Pour commémorer le cinquantenaire du décès de Le Corbusier, le laboratoire AlICe a choisi de s'intéresser aux six projets de l'oeuvre belge de l'architecte, avec le support de la Fondation Le Corbusier à Paris. Pour plusieurs de ces projets, il s'agit d'une première modélisation numérique, ce qui permettra de mieux faire connaître auprès d'un large public les projets de papier, souvent assez avancés, et d'avoir une source complémentaire dans l'étude de ces projets. D'autres projets ont, en revanche, déjà fait l'objet de nombreuses études. Il s'agit alors de trouver de nouveaux angles d'attaque, de nouveaux points de vue, ou de proposer une synthèse de la connaissance existante qui pourra trouver dans le média numérique une nouvelle forme de diffusion. Après une brève présentation des six projets belges de Le Corbusier, cet article propose de relater quelques enjeux méthodologiques précis de l'analyse architecturale par les moyens graphiques qui anticipent la création d'un court-métrage, et de mettre en évidence la connaissance que ce processus est susceptible d'apporter sur l'oeuvre belge du grand architecte.

\section{Le Corbusier et la Belgique}

Outre la maison Guiette (1926), seule réalisation de Le Corbusier encore présente sur le sol belge, l'œuvre corbuséenne en Belgique est surtout marquée par sa participation à l'exposition universelle de 1958de Bruxelles, avec le Pavillon Philips et le spectacle multimédia Le Poème Electronique, de lui-même et d'Edgar Varèse ${ }^{1}$. L'œuvre sera détruite après l'exposition. C'est d'ailleurs le seul projet après-guerre de Le Corbusier en Belgique. Les autres manifestations de son travail belge concernent l'architecture domestique, l'architecture et le mobilier d'exposition, et un plan d'urbanisme. Ces projets se compressent en une décennie, entre 1929 et 1939. Il s'agit de la maison Canneel à Bruxelles (1929), le plan pour la rive gauche à Anvers (1933), le pavillon du jeune homme pour l'exposition à Bruxelles en 1935, et le Pavillon de l'eau pour l'exposition de l'eau à Liège (1939). Ces quatre projets ne sont pas réalisés, et les informations d'archives pour ces projets diffèrent profondément d'un projet à l'autre.

Quelques années après la réalisation de la maison Guiette à Anvers, Jean-Jacques Canneel lui confie le projet d'une maison à Bruxelles, signalée dans le premier tome de L'œuvre complète comme « Maison pour M. X à Bruxelles $»^{2}$. Le projet de la maison reste au stade de l'avant-projet, et a fait l'objet d'une étude de Marc Dubois

\footnotetext{
${ }^{1}$ Petit, Jean (Ed.): Le Poème Electronique - Le Corbusier. Paris : Editions de Minuit, 1958. ; Treib, Marc: Space calculated in seconds. New Jersey : Princeton University Press, 1996.

${ }^{2}$ Boesiger, Willy; Storonov, Oscar: Le Corbusier et Pierre Jeanneret. Oeuvre Complète. 1910 - 1929. Zürich: Editions Girsberger, 1943. pp. 204-205.
} 
dans le cadre du projet réalisé sur une autre parcelle, en 1931, avec L.H. De Koninck ${ }^{3}$ (bâtiment démoli en 1969). Les documents d'archives disponibles sont surtout les plans, coupes et façades de l'avant-projet, ainsi que les croquis des espaces intérieurs et extérieurs. Presque tous ces documents se retrouvent dans L'œuvre complète. Il s'agit d'une maison avec une typologie très belge de parcelle peu large et très profonde, dans un nouveau quartier bruxellois, qui imposait de construire en hauteur. Le programme initial souhaité par Jean Canneel décrit des espaces de vie au rez-de-chaussée, en interaction avec le jardin, une chambre à coucher, et une bibliothèquebureau en relation avec le toit-terrasse. Or, le projet de Le Corbusier néglige complètement ces demandes, en proposants des espaces de vie traversants, et, au deuxième étage, en ajoutant une piscine en relation avec la chambre à coucher du premier étage. Les espaces domestiques se trouvent également au premier étage, côté rue. Contrairement à l'escalier conçu pour la Maison Guiette à Anvers, Le Corbusier prévoit un double escalier en forme d'amande en plan, séparant ainsi domestique et propriétaires, mais créant également un objet sculptural qui devient la colonne vertébrale de la maison. Le projet a donc tout intérêt à être étudié de plus près, ce qui lui permet d'être inséré dans les études de l'évolution des projets domestiques et des maisons type Citrohan de Le Corbusier à cette période.

La maison Guiette, quant à elle, a été conçue en 1926, et a fait l'objet d'une minutieuse restauration par Georges Baines en 1987, parallèlement à la construction par ce dernier d'une extension à gauche de la maison. Suite à la restauration de la maison et à l'exposition sur Le Corbusier à Anvers cette même année, et aussi grâce à la renommée de son propriétaire le peintre René Guiette, la maison, ses principes, et l'historique de conception, sont bien documentés et discutés ${ }^{4}$. Révéler un autre point de vue que celui issu des approches et du matériel photographique existants, comme les photographies canoniques utilisées par Le Corbusier et les photographies de famille de René Guiette, toutes en noir et blanc, était donc nécessaire. La polychromie de la restauration étant inaccessible étant donné le statut privé de la maison, il était intéressant de restituer en 3D cette polychromie, en particulier pour rendre compte du vécu de la maison par la famille Guiette perceptible dans cette série de photos. Plus qu'une restitution des intérieurs des années 1940, il s'agit de donner des clefs de lecture pour une compréhension des aménagements des espaces, en incluant évidemment les choix de peintures de René Guiette pour sa propre maison.

Le plan de la rive gauche d'Anvers, réalisé pour le concours Imalso en 1933, est d'une part une application parfaite de la Ville Radieuse de Le Corbusier, comme lui-même en témoigne dans la publication homonyme de 1931, et d'autre part une remise en question de certains principes de ce plan de la Ville Radieuse, suite à l'apport extérieur de Huib Hoste et Fé Louquet, collaborateurs depuis la Belgique pour le concours. Ce concours fut l'objet de plusieurs publications, où le projet de Le Corbusier est, malgré l'accueil mitigé qu'il a reçu du jury, largement étudié ${ }^{5}$. Ces études ont pour objet la mise en contexte historique du concours, l'inscription du plan

\footnotetext{
${ }^{3}$ Dubois, Marc: “Le Corbusier \& Louis Herman De Koninck. La maison Canneel 1929-1931”. In Burniat, Patrick (Ed.): Le Corbusier et la Belgique (Les Rencontres de la Fondation Le Corbusier). Bruxelles : CFC-Editions, 1997. pp.63-78.

${ }^{4}$ Baines, Georges; Spitaels, Els: Le Corbusier te Antwerpen : De Woning Guiette. Antwerpen : Hoger Architectuurinstituut van het rijk-stad Antwerpen, 1987. ; Schraenen, Guy: Les Peupliers. Maison Guiette = Huis Guiette. Le Corbusier 1926. Brussel : Sint-Lukasarchief, 1987.; Malliet, Anne: "Le Corbusier huis Guiette gerestaureerd". En $M \& L$. Novemberdecember 1987, N6, 1987. pp. 49-60. ; Prelorenzo, Claude (Ed.): La conservation de l'œuvre construite de Le Corbusier (Rencontres de la Fondation Le Corbusier). Paris : Fondation Le Corbusier, 1990. ; Burniat, Patrick (Ed.): Le Corbusier et la Belgique, op. cit.; De Heer, Jan: The Architectonic Colour: Polychromy in the Purist Architecture of Le Corbusier. Rotterdam: Nai010 Publishers, 2009.; Baines, Georges: "Du « système Dom-Ino » au «type Citrohan » : la maison Guiette à Anvers ”, En Massilia, 2011. pp. 50-81.

5 Commers, Ronald; Lombaerde, Piet: Le Corbusier en de Linkeroever te Antwerpen. Antwerpen: Hoger Architectuurinstituut van het Rijk (H.A.I.R.), 1987. ; Lombaerde, Piet: "Les projets pour la Rive Gauche d'Anvers, ou la Ville Radieuse en question”. In Burniat, Patrick (Ed.): Le Corbusier et la Belgique, op. cit. pp. 149-174.
} 
pour la rive gauche dans les plans urbanistiques de Le Corbusier, et l'apport des architectes belges dans le processus d'élaboration du plan.

La moitié des projets conçus pour la Belgique sont des projets de pavillons d'exposition. Pour l'exposition internationale à Bruxelles en 1935, Charlotte Perriand, René Herbst et Louis Sognot réalisent l'espace d'habitation d'un jeune homme. Le Corbusier et Pierre Jeanneret n'interviennent que partiellement pour le dessin de certains éléments de mobilier. Quelques plans dans les archives de la Fondation Le Corbusier témoignent cependant d'une étape architecturale réflexive sur le projet.

Ensuite, en 1939, l'architecte dessinera le pavillon de la France à l'Exposition de l'eau à Liège, également appelé le Pavillon de l'eau. Ce projet se caractérise par une structure en pylônes et cadres d'acier. Dans les quatre carrés des cadres se trouve une toile tendue en paraboloïde hyperbolique. La manière de tendre la toile fait référence au Pavillon des Temps Nouveaux de l'exposition universelle à Paris de 1937, et, comme le notent la plupart des études, il s'y trouve les prémisses du pavillon de Zurich pour Heidi Weber conçu en 1963 et achevé après la mort de Le Corbusier en $1967^{6}$. Différentes versions du projet du pavillon de l'eau existent, mais les informations restent très parcellaires et ne permettent pas de dépasser le stade d'un projet-croquis. Par contre, la plupart des documents d'archives sont des documents graphiques et non pas techniques, ce qui conduit à établir un propos analytique en concordance avec ce type de documents, en assumant les incertitudes.

Le dernier projet de Le Corbusier pour la Belgique, réalisé celui-ci, mais ensuite démoli, est le Pavillon Philips de l'Exposition universelle de Bruxelles en 1958. C'est aussi le dernier projet de pavillon de l'architecte.

Cette exposition est la première du genre à avoir lieu après la seconde guerre mondiale. Cette particularité, ainsi que la spécificité de l'œuvre conçue par Le Corbusier en collaboration avec Iannis Xenakis pour le pavillon, et Edgar Varèse pour le spectacle multimédia Le Poème Electronique, confère à l'objet un intérêt particulier dont témoignent plusieurs études monographiques qui se focalisent autant sur l'aspect audiovisuel, la conception et l'exécution de la structure, que sur la présence du bâtiment au sein de l'Exposition ${ }^{7}$. Le projet VEP (Virtual Electronic Poem) propose, par la réalité virtuelle, une reconstitution visuelle et auditive convaincante de l'expérience sensorielle du l'intérieur du pavillon ${ }^{8}$. Ce projet donne ainsi lieu à une expérience nouvelle, différente du film officiel tourné en 1958, et se situe plutôt en résonance avec les photographies d'époque de Lucien Hervé qui présentent les têtes des visiteurs faces aux grandes projections sur les murs courbes du pavillon. Un travail effectué par des étudiants ne peut prétendre rivaliser avec un projet d'une telle ampleur. L'approche sera donc plutôt celle de la synthèse rigoureuse mais didactique.

\footnotetext{
${ }^{6}$ Baiwir, Jean-Claude: "Liège 1939: d'un pavillon d'exposition à l'autre" In Burniat, Patrick (Ed.): Le Corbusier et la Belgique, op. cit. pp. 199-210. ; Dumont D’ayot, Catherine; Benton, Tim: Le pavillon de Le Corbusier pour Zurich : modèles et prototype d'un espace d'exposition idéal. Zurich: Lars Müller Publishers, 2013.

${ }^{7}$ Petit, Jean (Ed.): Le Poème Electronique, op. cit..; Treib, Marc: Space calculated in seconds. New Jersey : Princeton University Press, 1996.; Sterken, Sven: Iannis Xenakis, ingénieur et architecte. Director: Bart Verschaefel. Gent Universiteit, 2004. ; Devos, Rika: Modern at Expo 58. Discussions on post-war architectural representation. Director: Mil De Kooning. Gent Universiteit, 2008.

${ }^{8}$ Lombardo, Vicenzo and others: “A Virtual-Reality Reconstruction of Poème Electronique Based on Philological Research". En Computer Music Journal. “A Virtual Poème”. Summer 2009, N². Cambridge Ma: MIT Press, 2009. pp. 24-47.
} 


\section{Confrontation des sources au sein d'espaces graphiques communs : instauration d'hypothèses}

La recherche effectuée par les étudiants se base d'abord sur une étude bibliographique, sur le fond de plans numérisés ${ }^{9}$ ainsi que sur les archives de la Fondation Le Corbusier à Paris auxquelles ils ont eu accès. De ces documents de nature diverses (étude monographique, travaux de recherche, photos anciennes, plans de diverses étapes du projet, croquis, maquettes, archives écrites comme la correspondance ou des procès-verbaux de réunion, etc.) peut être extraite une description de taille et d'importance variables d'une partie ou de l'ensemble du projet étudié. Le premier enjeu d'une analyse par les moyens graphiques est de confronter les informations contenues dans ces différentes sources de façon à esquisser une ou plusieurs instances hypothétiques de la géométrie de l'objet étudié, correspondant à une époque ou plusieurs époques définies. Les sources bibliographiques et archivistiques sont donc triées, hiérarchisées, et classées suivant différents critères, puis sont confrontées au sein d'espaces graphiques ${ }^{10}$ communs de plus en plus détaillés : d'abord des vues géométrales et des dessins axonométriques, ensuite des maquettes d'étude, et enfin des modèles 3D numériques. Cette mise en commun des informations présentes dans diverses sources nécessite de résoudre toute une série de contradictions ou d'incohérences que ces sources mettent en exergue une fois qu'on les confronte. Certains détails doivent être interprétés parce que les documents d'archives n'en permettent qu'une compréhension parcellaire, d'autres - la plupart du temps les détails techniques - doivent être simplifiés lorsque leur description originale est trop précise pour que le niveau de détail du modèle soit cohérent et homogène. Enfin, certaines parties doivent être inventées car leur description est manquante, mais pour assurer l'harmonie visuelle et géométrique de l'ensemble, il y a nécessité de «combler les trous ». Cette invention se base néanmoins sur la compréhension de la totalité du projet étudié, et constitue une extrapolation de principes récurrents de l'architecture de Le Corbusier à des époques similaires. Des références peuvent donc être trouvées dans d'autres projets et transposées sur le bâtiment en question. Par exemple, l'étude portant sur la Maison Canneel (1929) propose une hypothèse de mise en couleurs des murs intérieurs qu'aucun document d'archives spécifique au projet ne permet d'étayer. Néanmoins, la compréhension des modes de mise en couleurs des maisons de type Citrohan est bien documentée ${ }^{11}$. La Maison Canneel étant apparentée à cette famille de projets, des principes de coloration peuvent en être extraits et transposés au projet bruxellois. Il en résulte une proposition dont on assume le statut extrêmement hypothétique, mais qui comporte une dimension exploratoire intéressante (1).

\footnotetext{
${ }^{9}$ Le Corbusier. Plans. 4 coffrets de 4 DVD Echelle-1 Fondation Le Corbusier

${ }^{10}$ Nous proposons de définir un espace graphique comme étant un univers représentationnel homogène, dans lequel coexistent une ou plusieurs figures caractérisées par un même mode projectif (géométral, perspectif, axonométrique), une même échelle, une même technique d'expression, et un même support matériel ou numérique. Un espace graphique peut être bidimensionnel (les modes projectifs) ou tridimensionnel (les maquettes). La mise en place de tels espaces permet de rassembler les informations géométriques issues de sources hétérogènes dans un document homogène, et ce à des fins de comparaison.

${ }^{11}$ Le Corbusier: Salubra, Claviers de couleur. Bâle, Editions Salubra, 1931. ; Schraenen, Guy: Les Peupliers. Maison Guiette = Huis Guiette, op cit. ; Prelorenzo, Claude: Le Corbusier et la couleur (Rencontres de la Fondation Le Corbusier). Paris: Fondation Le Corbusier, 1992. ; De Heer, Jan: The Architectonic Colour: Polychromy in the Purist Architecture, op. cit.
} 


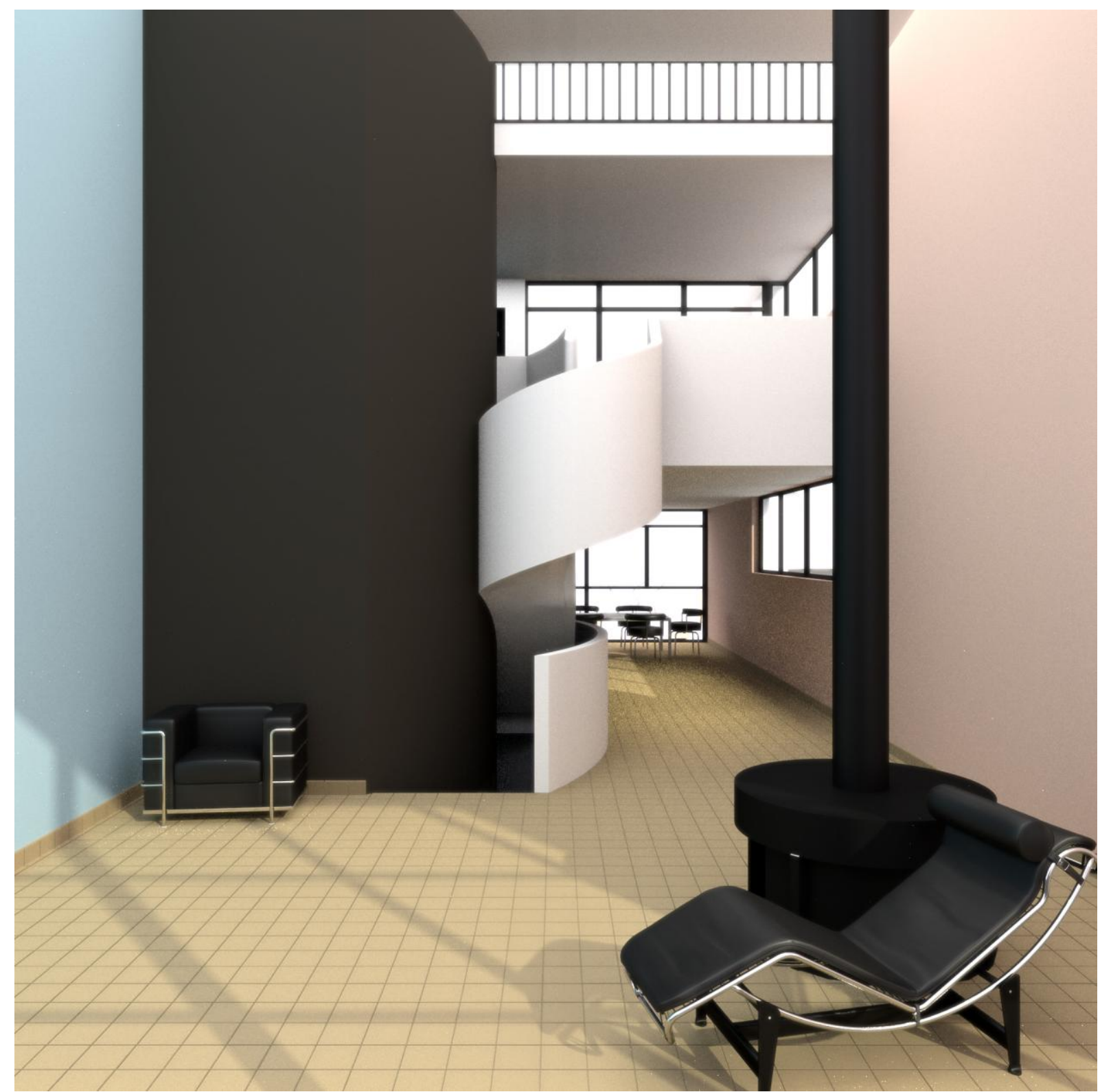

1. Maison Canneel (1929). Hypothèse de restitution, vue du salon et de la cage d'escalier.

L'interprétation ou l'invention sont également rendues nécessaires par l'incohérence fréquente des documents d'archives entre eux : certains éléments en plan, en coupe, en axonométrie, ou en maquette, sont différents, car issus de différentes étapes de la réalisation du projet, étapes parfois difficilement identifiables. Le modèle 3D entreprend alors de « résoudre » ces incohérences, tout en renseignant les choix effectués dans les documents du processus d'élaboration de l'analyse. Tout cela amène nécessairement à produire des objets graphiques $^{12}$ singuliers et interprétatifs, mais dont le processus d'élaboration reste traçable. Par conséquent, le modèle 3D issu du processus analytique propose seulement une ou plusieurs hypothèses ; plutôt que de prétendre se substituer à une réalité potentielle ou disparue, il se contente de relater un état de connaissance sur un projet dans un contexte particulier, et sur base de sources précises et identifiables.

\footnotetext{
${ }^{12}$ Nous proposons également de définir un objet graphique comme étant une figure d'un espace graphique, reprenant intégralement les caractéristiques de ce dernier.
} 
Cette démarche de confrontation des sources peut être observée dans l'analyse menée sur la Maison Canneel : les plans des différents niveaux sont ceux d'un avant-projet datant de 1929. Ils ne correspondent pas parfaitement entre eux lorsqu'on les superpose, et ne permettent pas réellement de reconstituer précisément les détails complexes et l'enchevêtrement spatial généré par la double circulation verticale et les courbes de la cage d'escalier, objet dominant et structurant dans la composition de la maison. Mis à part son registre formel extravagant, cette cage d'escalier se réfère à une typologie de circulation verticale et à un mode d'organisation du plan typiquement bruxellois, adapté à des parcelles étroites et profondes qui caractérisent le tissus urbain de la capitale belge. Bien que peu d'escaliers de projets de Le Corbusier semblent avoir une telle complexité formelle, certains projets sont clairement liés au projet Canneel; notons par exemple la double cage d'escalier de la Maison Cook à Boulogne-Billancourt (1926), le simple escalier de la Villa Stein-de Monzie (1927), et le pan de verre d'entrée de la villa Savoye (1929), dont le projet était à ce moment en cours. Plusieurs investigations dans divers espaces graphiques seront entrepris pour comprendre cet élément d'articulation de la Maison Canneel (2). Il en résultera un modèle 3D hypothétique qui se distance légèrement des sources originales, mais qui est tectoniquement cohérent.

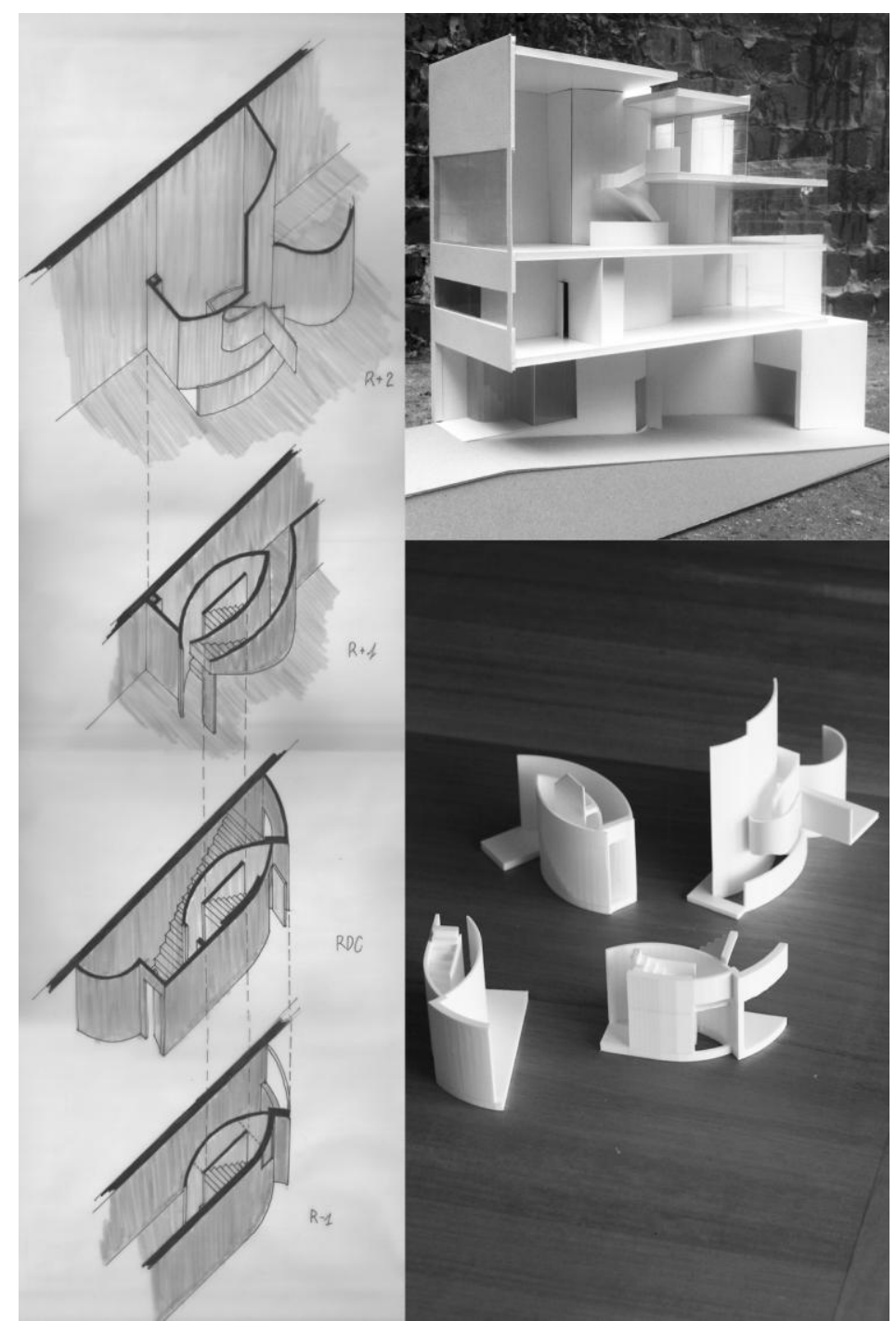

2. Maison Canneel (1929). Documents d'analyse graphique : maquette d'étude en carton et décomposition analytique de la cage d'escalier par l'axonométrie et l'impression 3D. 
Les archives relatives au Pavillon de l'Exposition de l'eau (1937) sont elles aussi très parcellaires et issues de versions du projet qui présentent des variations formelles et structurelles assez importantes. Les références bibliographiques sont également restreintes, le projet ayant fait l'objet de peu d'études. Il est donc nécessaire de comprendre l'approche de Le Corbusier dans d'autres projets de pavillons précédents, comme le Pavillon Nestlé (1928), contemporains comme le Pavillon des Temps Nouveaux (1937), ou ultérieurs comme le Pavillon de Zurich (1963), qui semble dériver directement du projet du Pavillon de l'eau ${ }^{13}$. Le modèle 3D résultant du processus analytique est hautement hypothétique et se contente d'évoquer les grands principes du projet, et seulement quelques détails de diverses versions de la structure avec un degré de certitude très relatif. Mais l'hypothèse inclut néanmoins une dimension exploratoire qui permet de suggérer l'univers spatial qu'aurait engendré le projet (3).

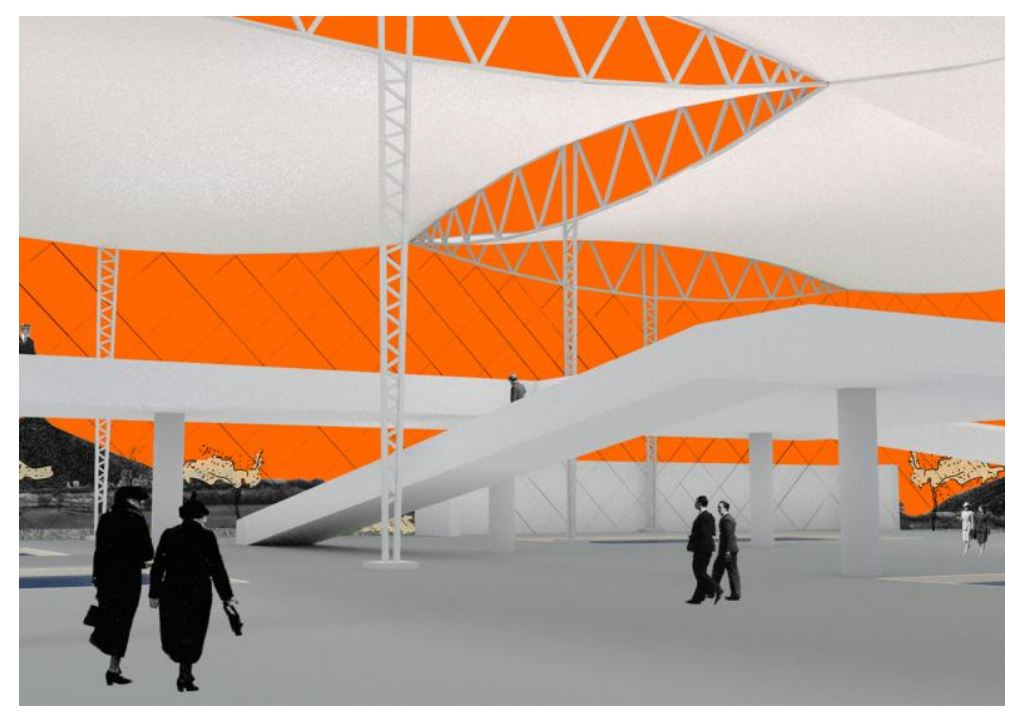

3. Pavillon de l'Exposition de l'eau (1939). Hypothèse de restitution de la spatialité intérieure du projet.

La Maison Guiette (1926) est le seul projet de Le Corbusier subsistant encore actuellement sur le sol belge, mais le bâtiment a subi des transformations importantes ainsi qu'une rénovation. Les étudiants se sont intéressés à l'intérieur de la maison tel qu'il est présenté dans une série de photographies datant approximativement de 1940, et ont tenté de restituer le projet tel qu'il était alors, avec la mise en couleurs des murs et le mobilier d'époque. Dans ce cas-ci, le bâtiment lui-même est source d'informations. L'hypothèse de restitution envisagée ici propose donc de confronter l'état actuel de la maison aux quelques photos dites officielles de Le Corbusier, devenues des vues canoniques, ainsi qu'aux photographies de famille réalisées par le peintre René Guiette lui-même. Bien que toutes ces photographies soient en noir et blanc, elles nous livrent des indications sur la manière dont la maison était vécue, sur l'ameublement, et sur les choix de tableaux exposés sur les murs dont Guiette était l'auteur. Dans l'étude de couleurs, la restitution des œuvres de ce peintre dans l'espace couleur corbuséen ${ }^{14}$ nous offre également des indications sur le choix de tableaux par rapport aux murs neutres et non-neutres. Le fait que

\footnotetext{
${ }^{13}$ Dumont d'Ayot, Catherine; Benton, Tim: Le pavillon de Le Corbusier pour Zurich : modèles et prototype d'un espace d'exposition idéal. Zurich: Lars Müller Publishers, 2013.

${ }^{14}$ En ce qui concerne la relation entre l'espace et les couleurs corbuséennes en tant que réceptacle d'une collection d'art, un parallèle peut être établi avec la Villa La Roche. La même année que la réception de la Maison Guiette a lieu la réception de la Villa La Roche. A cette occasion, Raoul La Roche note : "Je vous avais commandé un « cadre pour ma collection ". Vous me fournissez un «poème en murs ». Qui de nous deux a été le plus fautif ?” (FLC, doc 506 bis, 24 mai 1926).
} 
l'artiste soit lui même intervenu dans le choix des couleurs effectué par Le Corbusier donne de bonnes indications sur la manière dont le peintre envisageait les teintes de sa maison en concordance avec son travail ${ }^{15}$. Les informations issues du bâtiment lui-même, celles issues des diverses photographies, ainsi que les études relatives aux couleurs des espaces corbuséens sont confrontées dans un même modèle 3D. Le document graphique ainsi obtenu propose une hypothèse de restitution en images de synthèse qui permet de se déplacer dans l'espace tel qu'il était supposé être aménagé à l'époque, en partant des points de vue identiques à ceux des photographies iconiques de 1940 (4 et 5).

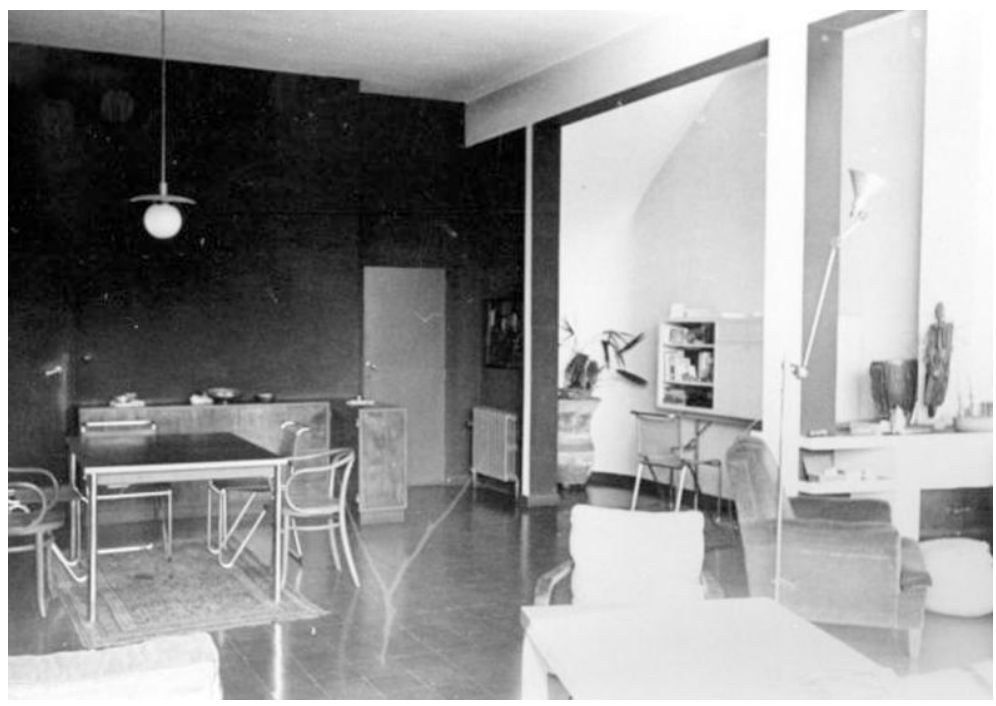

4. Maison Guiette (1926). Vue de la salle à manger vers 1940.

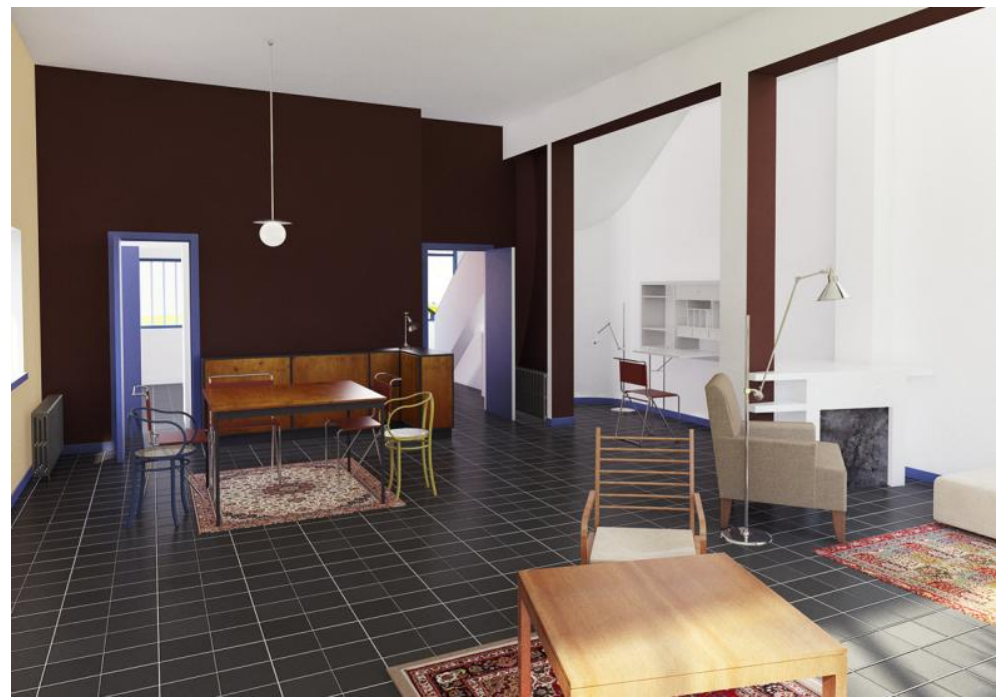

5. Maison Guiette (1926). Hypothèse de restitution colorisée de l'espace de la salle à manger aux alentours de 1940.

\footnotetext{
${ }^{15}$ De Kerckhove d'Ousselghem, Manoela; Goyens de Heusch, Serge: René Guiette. Antwerpen: Mercatorfonds, 1991. ; Schraenen, Guy: Les Peupliers. Maison Guiette = Huis Guiette, op. cit. ; De Heer, Jan: The Architectonic Colour: Polychromy in the Purist Architecture, op. cit.
} 


\section{Analyse architecturale par hybridation des techniques graphiques}

La compréhension analytique et critique du contexte historique, théorique, social et politique est une composante fondamentale pour la connaissance d'un bâtiment, ainsi que pour charpenter le propos d'un court-métrage architectural. Néanmoins, cette problématique ne sera pas abordée ici, ce type d'étude ne procédant pas par les moyens graphiques qui sont le sujet du présent article. La conception architecturale quant à elle, s'élabore bel et bien par les moyens graphiques. Comme l'a remarqué Robin Evans, l'architecte ne manipule l'objet de sa création que par l'intermédiaire de représentations graphiques, à l'inverse des peintres et des sculpteurs qui sont en contact direct avec l'objet de leur création ${ }^{16}$. Ceci confère aux techniques graphiques un énorme potentiel génératif. Lors du processus de conception, les informations voyagent d'un document à l'autre en s'adaptant à diverses techniques de représentation. Lors de ces transferts successifs, des éléments s'évanouissent et d'autres apparaissent, ce qui a pour conséquence de faire évoluer l'objet architectural traité ${ }^{17}$. L'évolution du processus de conception architecturale par les moyens graphiques, la transformation du propos par le dialogue entre les artefacts médiateurs, peuvent également être pris en compte lors du processus d'analyse formelle d'un bâtiment. En effet, tant la conception que l'analyse visent à établir un discours architectural par une décomposition et une recomposition successives des formes, et ce dans le but de manipuler la géométrie dans des espaces graphiques abstraits. Dès lors, l'analyse peut être comprise comme une forme de rétro-conception, qui opère par un dialogue de moyens de représentation similaires à ceux de la conception ${ }^{18}$, et pour laquelle l'usage de techniques graphiques variées relève de la même capacité générative que celle décrite par Evans. L'analyse par les moyens graphiques se focalise donc sur les aspects formels, spatiaux et tectoniques. Pour investiguer tel ou tel autre aspect de l'objet architectural, il s'agit d'utiliser chaque média, traditionnel ou numérique, chaque mode de projection canonique, en fonction de ses spécificités. Ensuite, il convient de faire dialoguer entre eux les objets graphiques hétérogènes ainsi obtenus. Les informations contenues dans un document d'un type de média particulier vont soulever de nouvelles questions lorsque l'on décide de les transposer dans un autre média, et ainsi de suite ${ }^{19}$. Lors du passage d'un média bidimensionnel à un média tridimensionnel, certaines informations apparaissent - ou doivent être inventées pour assurer la cohérence visuelle et géométrique de l'ensemble (voir le point 3 du présent texte) - ce qui a pour conséquence d'enrichir la connaissance géométrique de l'objet étudié. A l'inverse, lors du passage d'un média tridimensionnel à un média bidimensionnel, certaines informations sont perdues, mais à l'avantage d'une plus grande clarté de lecture d'un concept particulier ${ }^{20}$. Finalement, la construction générale du court-métrage fait également appel aux techniques graphiques : le storyboard est un document de synthèse sujet à de nombreuses modifications. Il reprend des schémas simplifiés des vues mises au point dans le processus analytique, les articule entre elles et les combine aux autres médias qui composeront le

\footnotetext{
${ }^{16}$ Evans, Robin: "Translations from Drawing to Building”. En Translations from Drawing to Building and Other Essays. London: Architectural Association Publications, 1997. p.156.

${ }^{17}$ Ce caractère sériel inhérent à la représentation architecturale - aussi appelé propriété de secondarité - a également été étudié par Philippe Boudon et Frédéric Pousin dans Boudon, Philippe; Pousin, Frédéric: Figures de la conception architecturale: manuel de figuration graphique. Paris: Editions Dunod, 1988.

18 A propos de la dimension active et exploratoire de l'analyse architecturale par les moyens graphiques, voir Jenkins, Eric J: Drawn to Design. Analysing Architecture Through Freehand Drawing. Basel: Birkhäuser, 2013.

${ }^{19}$ Les enjeux méthodologiques d'une telle approche sont développés plus exhaustivement dans Derycke, Denis; Lo Buglio, David: "Education in Architectural Analysis through Hybrid Graphical Means: A Setup for Critical Thinking". En Design \& Graphic Palimpsest. Atlanta GA: Southern Polytechnic State University, 2014. pp. 306-12.

${ }^{20}$ En réaction à la trop grande densité d'informations simultanées imposée par les modèles 3D numériques, Joël Sakarovitch souligne la capacité d'énonciation des modes projectifs traditionnels : "parfois en dire peu c'est en dire beaucoup". Dans Sakarovitch, Joël: Épures d'architecture: de la coupe des pierres à la géométrie descriptive XVIe-XIXe siècles. Basel: Birkhäuser, 1998.
} 
film. Il rassemble un maximum d'informations pour anticiper la création du film, permet de rendre le processus traçable et aussi d'identifier les sources (6).

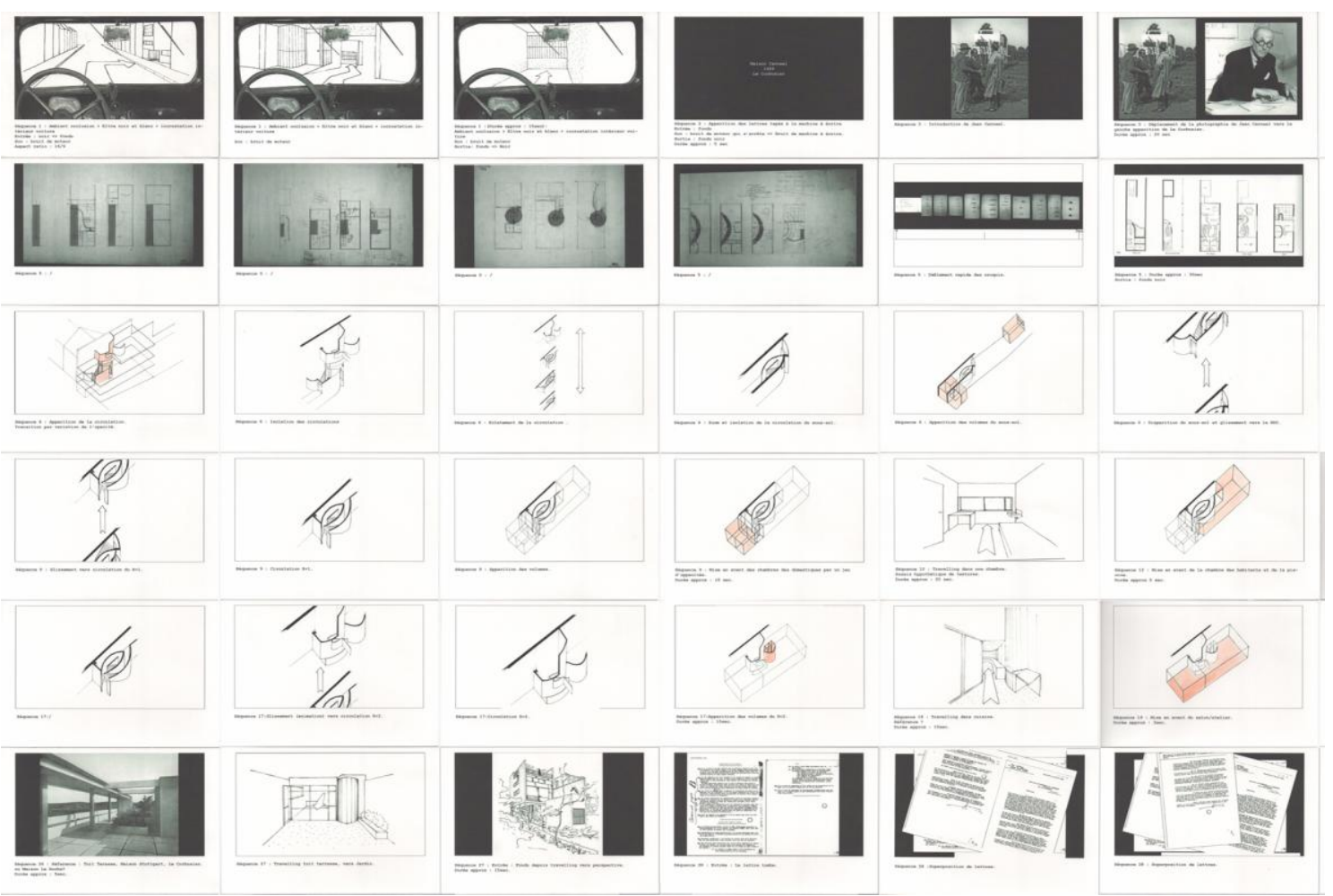

6. Maison Canneel (1929). Transcription du propos analytique dans un storyboard anticipant la création d'un court-métrage.

Si les techniques graphiques ont une influence évidente sur les concepts architecturaux manipulés, cette influence peut parfois devenir problématique lors de l'utilisation de logiciels sophistiqués dédiés à la conception architecturale. En effet, ces logiciels dits «métier» “contiennent une théorie implicite de la conception, jamais d'ailleurs explicitée $^{, 21}$. Pour profiter des apports des logiciels 3D tout en conservant une réflexion architecturale relativement autonome, les études par modélisations 3D sont systématiquement effectuées sur des programmes indépendants du domaine de l'architecture, comme par exemple le logiciel open source Blender ${ }^{22}$.

L'apport de connaissances d'une analyse par hybridation des techniques graphiques apparait de manière assez évidente dans le travail effectué sur la Maison Canneel. L'investigation commence par une compréhension rapide de l'objet et de sa volumétrie par une maquette d'étude en carton à a $1 / 50^{\text {ème }}$ (2). La maquette permet de dégrossir rapidement la composition et les articulations formelles globales, tout en évitant certaines questions géométriques trop précises face auxquelles le modèle $3 \mathrm{D}$ force à se positionner. Parallèlement, des croquis axonométriques décomposent et analysent l'élément complexe caractérisant le projet : la cage d'escalier (2). Le travail est ensuite affiné par la modélisation 3D qui permet, dans un second temps, un travail plus précis et plus détaillé que les médias traditionnels, ainsi que la possibilité de tester facilement des variantes sur certaines hypothèses. Pour chaque niveau, les éléments de la cage d'escalier sont extraits du modèle 3D de manière à

\footnotetext{
${ }^{21}$ Michel Léglise se basant sur une étude de Ronald Hamel dans Léglise, Michel: "Ordinateurs dans l'apprentissage de la conception : mental et instrumental”. En Computerised Craftsmanship. Paris: eCAADe Conference Proceedings, 1998. p.139.

${ }^{22}$ http://www.blender.org/ (consulté le 07 juin 2015).
} 
correspondre à ceux étudiés dans les croquis axonométriques, puis imprimés en 3D, étage par étage, et enfin assemblés (2). Revenir du modèle 3D ou des modes projectifs traditionnels vers les moyens de représentation tactiles permet une compréhension plus approfondie de certains éléments que les modes projectifs ne suffisent pas toujours à décrire clairement et efficacement. L'impression 3D s'avère particulièrement appropriée dans ce genre de démarche. Cette représentation de la cage d'escalier de la Maison Canneel, ainsi extraite de son contexte, prend une dimension sculpturale et constitue un regard analytique, didactique, mais aussi interprétatif de l'oeuvre. De la même manière que les axonométries plafonnantes d'Auguste Choisy créaient un univers fictionnel éloigné de l'objet auquel elles se référaient ${ }^{23}$, l'objet-sculpture résultant de l'impression en 3D de la cage d'escalier « raconte » la maison Canneel d'une autre manière. Partant d'une base analytique et objective, l'objet ne montre plus directement le projet mais évoque plutôt la richesse du registre formel de Le Corbusier (7).

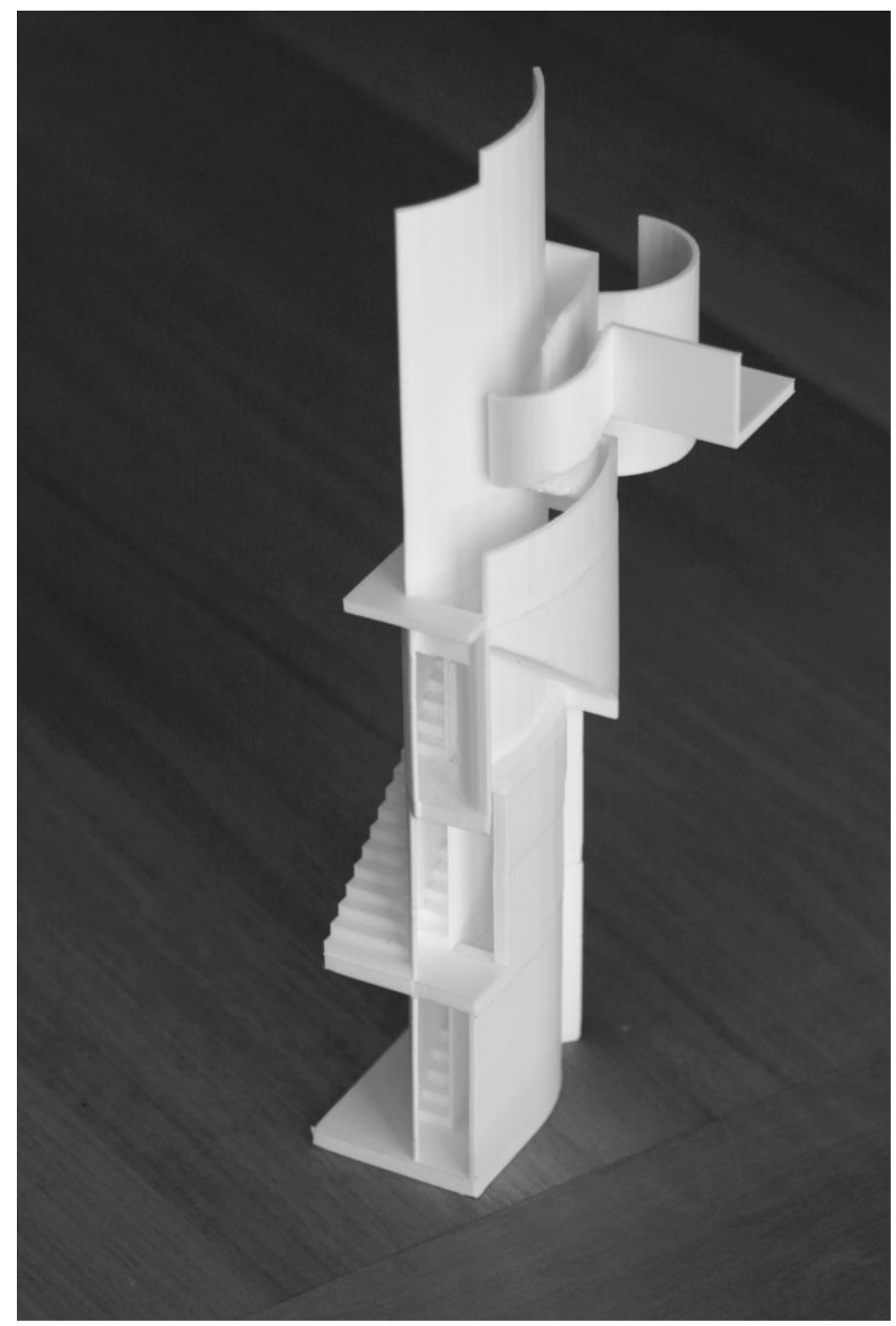

7. Maison Canneel (1929). Analyse et hypothèse de restitution de la cage d'escalier par l'impression 3D.

\footnotetext{
${ }^{23}$ Mandoul, Thierry: “Une histoire dessinée”. En Entre raison et utopie. L'Histoire de l'Architecture d'Auguste Choisy. Wavre: Mardaga Editions, 2008. pp. 111-162.
} 


\section{Choix de représentation graphique et qualification des hypothèses}

Comme expliqué précédemment, un choix pertinent des moyens de représentation graphique permet d'appuyer le processus analytique et exploratoire d'un bâtiment. Cette compréhension de la représentation architecturale se poursuit dans la manière d'exprimer les concepts analysés en vue de la production des images du court-métrage. Le niveau de détail, l'échelle, la couleur, le mode projectif, l'univers graphique, etc., participent à l'élaboration du message. Malgré l'extraordinaire diversification récente des moyens d'expression à la disposition des architectes, la compréhension des codes de la représentation en vigueur depuis des siècles reste un canevas de référence incontournable. Par exemple, il est important de transposer dans le média numérique 3D une différence cognitive de base que l'utilisation des programmes a tendance à masquer : la perspective informe sur ce que l'on perçoit d'un objet et l'axonométrie nous informe sur ce que l'on sait d'un objet ${ }^{24}$. Parce qu'elle évacue la question de l'observateur incarné, l'axonométrie est appropriée pour une expression analytique et décontextualisée de l'objet. La perspective conique, qui cherche l'osmose avec la perception visuelle, convient bien pour proposer des hypothèses de la perception spatiale que pouvait avoir un observateur se déplaçant dans l'espace intérieur ou extérieur du bâtiment étudié. Les choix de représentation qualifient également le niveau de certitude qu l'on peut avoir par rapport aux hypothèses de restitution que l'on propose. Les modèles 3D et l'image de synthèse contemporains permettent d'obtenir des rendus photoréalistes qui peuvent donner l'illusion d'une expérience spatiale vécue d'un projet, la différence entre des images filmées et des images numériques devenant presque impossible à établir. Si ce mode de rendu peut s'avérer approprié dans certains cas, il peut également créer des ambiguïtés, notamment lorsque le niveau de connaissances sur l'objet étudié ne permet pas de créer le substitut de réalité devenu accessible à l'aide des images de synthèse actuelles. Dès lors, des modes de représentation plus symboliques sont appropriés, utilisant à bon escient les codes de la représentation architecturale, puisant dans des registres expressifs variés, et cherchant plus à évoquer un univers possible qu'à se substituer à une réalité par trop hypothétique. Comme le rappelle Antoine Picon, "le projet n'est jamais une configuration fermée"25, et ajoutant ensuite : "le dessin d'architecture a toujours été au moins autant destiné à évoquer un espace de possibles que voué à la fixation de la morphologie du projet ${ }^{\text {} 26}$. Ceci se vérifie lorsqu'on s'intéresse à des projets dont l'existence se cantonne à quelques planches dans un fond d'archives. Dans ce cas, des telles considérations doivent guider tant la compréhension que l'on peut avoir des dessins que l'on observe que l'élaboration des représentations que l'on produit pour exprimer ces observations. En laissant une place pleinement assumée à une interprétation qui est clairement comprise comme telle, la dimension exploratoire des nouvelles représentations permettent de faire émerger un nouveau questionnement. Le degré de certitude des informations présentées est accessible une fois que l'on confronte les représentations produites aux diverses sources que ces représentations tentent de rassembler au sein d'un même objet graphique.

\footnotetext{
${ }^{24}$ Schneider, Bernhard: "Perspective Refers to the Viewer, Axonometry Refers to the Object". En Drawing as Medium of Abstraction. Daidalos - Berlin Architectural Journal, September 1981, №1. Berlin: Wilhem Krümpelmann, 1981. pp. 81-95.

${ }^{25}$ Picon, Antoine: “Architecture et mémoire numérique”. En Archigraphy. Paris: Infolio, 2007. p.61.

${ }^{26} I d$.
} 
La Maison Canneel, restée au stade de l'avant-projet, soulève des questions de représentation intéressantes lorsqu'il s'agit d'en exprimer une hypothèse de restitution par l'image numérique 3D. Deux dessins originaux de Le Corbusier, l'un du rez-de-chaussée de la façade avant et l'autre présentant le bâtiment tel que vu depuis l'intérieur de l'îlot, ont été réinterprétés lors de la réalisation du court-métrage sur ce projet. Le premier apport de l'image numérique est de donner une matérialité plus consistante à l'objet étudié par la simulation de la lumière sur les volumes puristes de la maison. Ensuite, le projet est situé à l'endroit où il était supposé voir le jour, et entouré d'un environnement bâti schématique, tel qu'il pouvait être aux alentours de 1940. Les informations disponibles ne permettant pas d'aller beaucoup plus loin dans l'hypothèse, l'environnement végétal dessiné par Le Corbusier est extrait des dessins et transposé tel quel dans le modèle 3D. Cette démarche vise à souligner le statut intermédiaire de ces images : un supplément de connaissance est apporté à l'objet tel qu'il se trouve décrit dans les archives, tout en manifestant le statut hypothétique des propositions, et en ne se détachant que partiellement des vues originales (8 à 11).

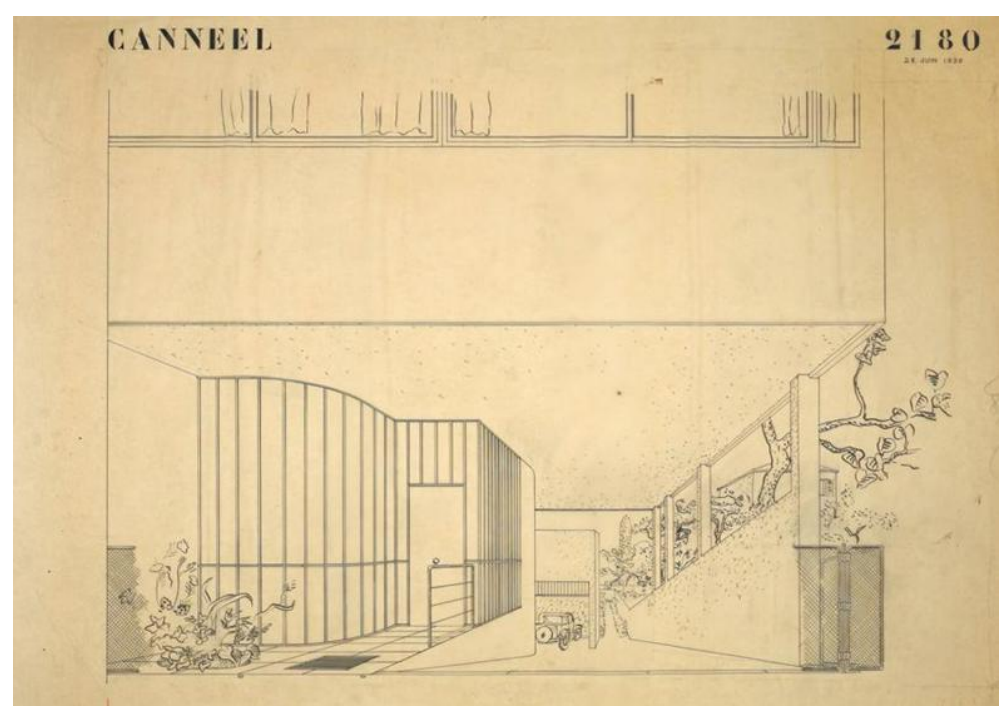

8. Maison Canneel (1929). Perspective de l'entrée et du rez-de-chaussée.

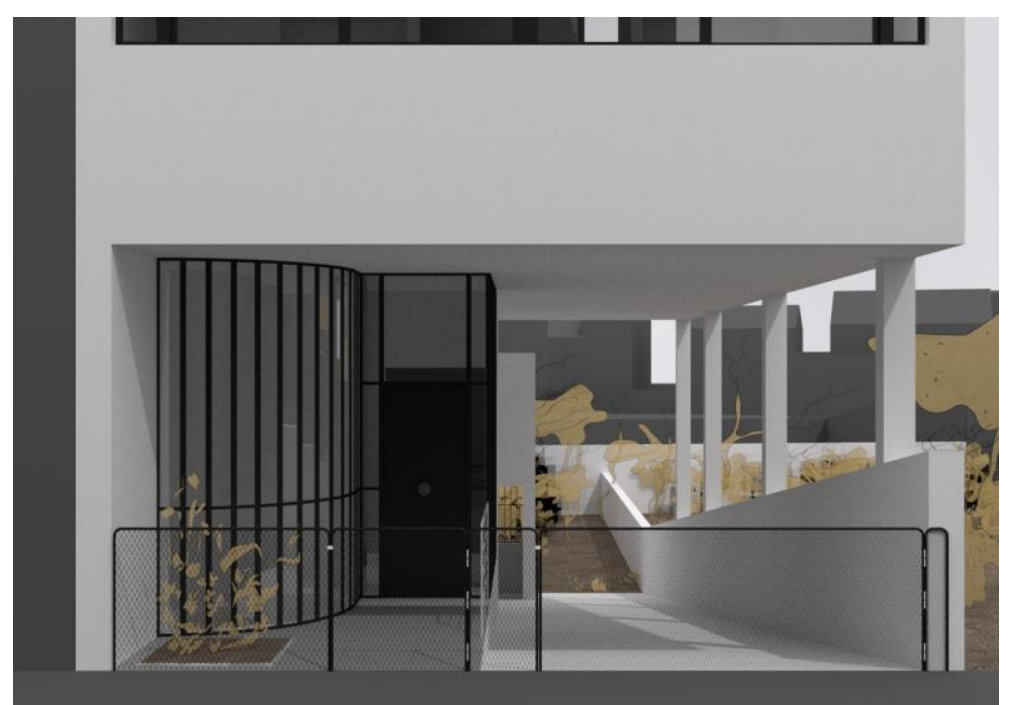

9. Maison Canneel (1929). Hypothèse de restitution de la perspective de l'entrée et du rez-de-chaussée. 


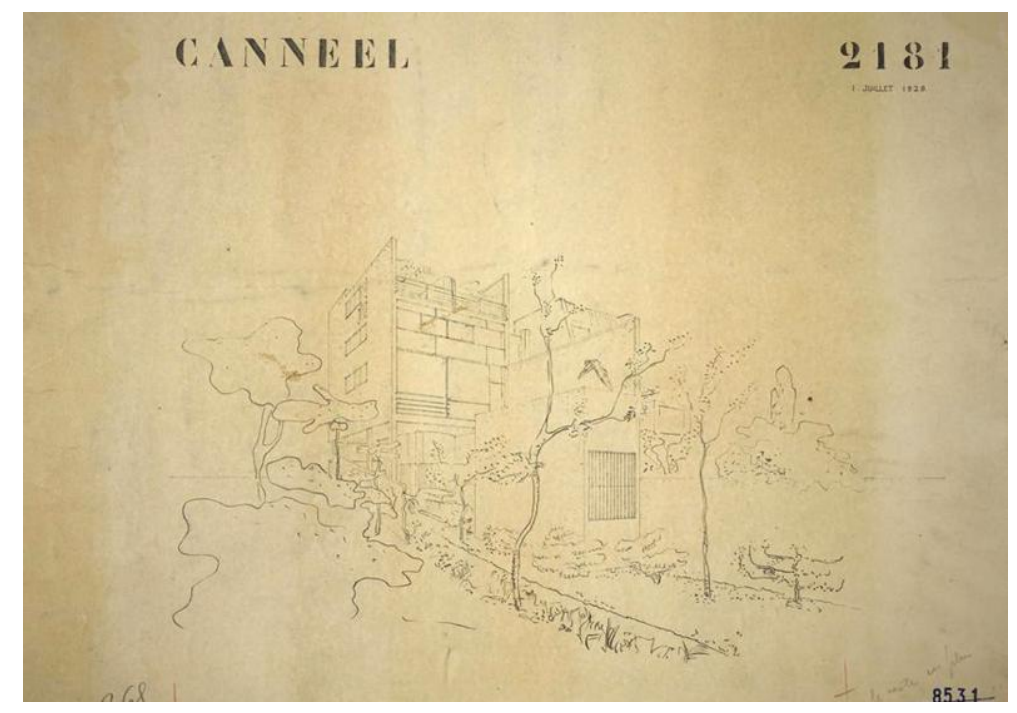

10. Maison Canneel (1929). Perspective de la maison depuis l'intérieur de l'îlot.

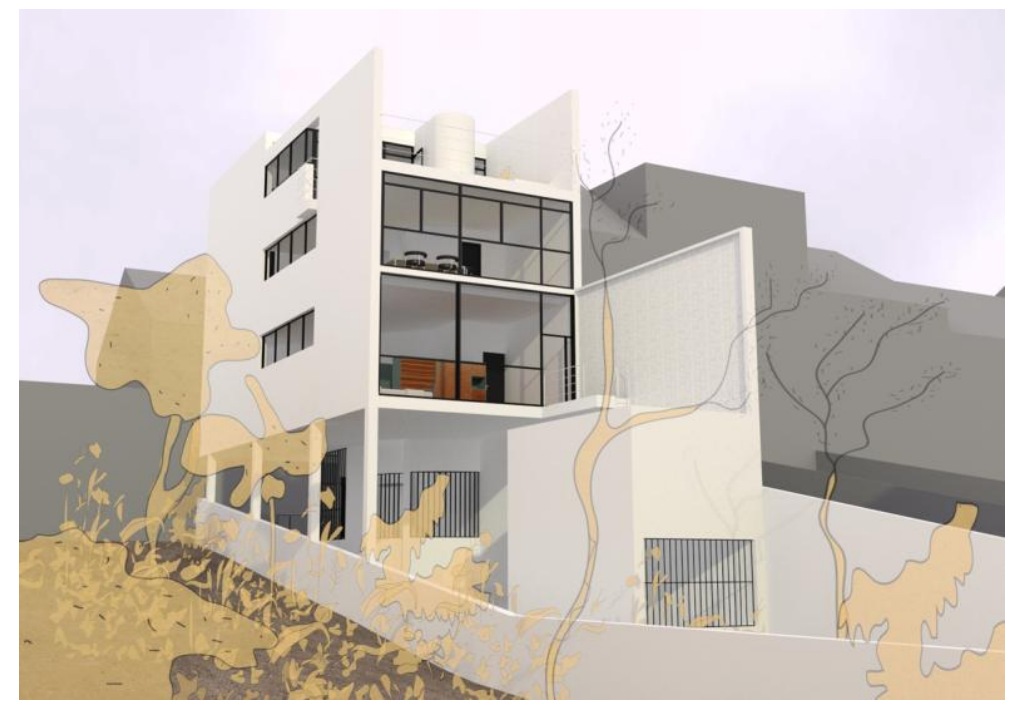

11. Maison Canneel (1929). Hypothèse de restitution de la perspective de la maison depuis l'intérieur de l'îlot.

Pour le dessin représentant la vue du salon et la cage d'escalier sculpturale, la démarche est similaire, tout en s'autorisant une légère incursion dans le domaine du réalisme. Les archives ne contiennent aucune indication concernant les matériaux de sol, les finitions et les couleurs des murs, sujet pourtant très important dans le travail de l'architecte à cette époque. Sur base du travail de Jan de $\mathrm{Heer}^{27}$, une hypothèse de mise en couleurs est alors proposée (1), et signalée comme telle dans le film. Dans le dessin original (12) apparaissent par contre des éléments de mobilier dessinés par Le Corbusier et Charlotte Perriand. Ces meubles sont modélisés en 3D et replacés dans l'espace aux mêmes endroits que sur les croquis. L'image numérique résultante donne à l'espace une impression de matérialité plus importante, mais son faible niveau de détail général la met hors de toute ambiguité. Ces trois dessins de Le Corbusier, une fois réinterprétés en images numériques, servent de point de départ à trois travellings qui permettent de profiter pleinement de la spatialité proposée par l'hypothèse de restitution.

\footnotetext{
${ }^{27}$ De Heer, Jan: The Architectonic Colour: Polychromy in the Purist Architecture, op. cit.
} 


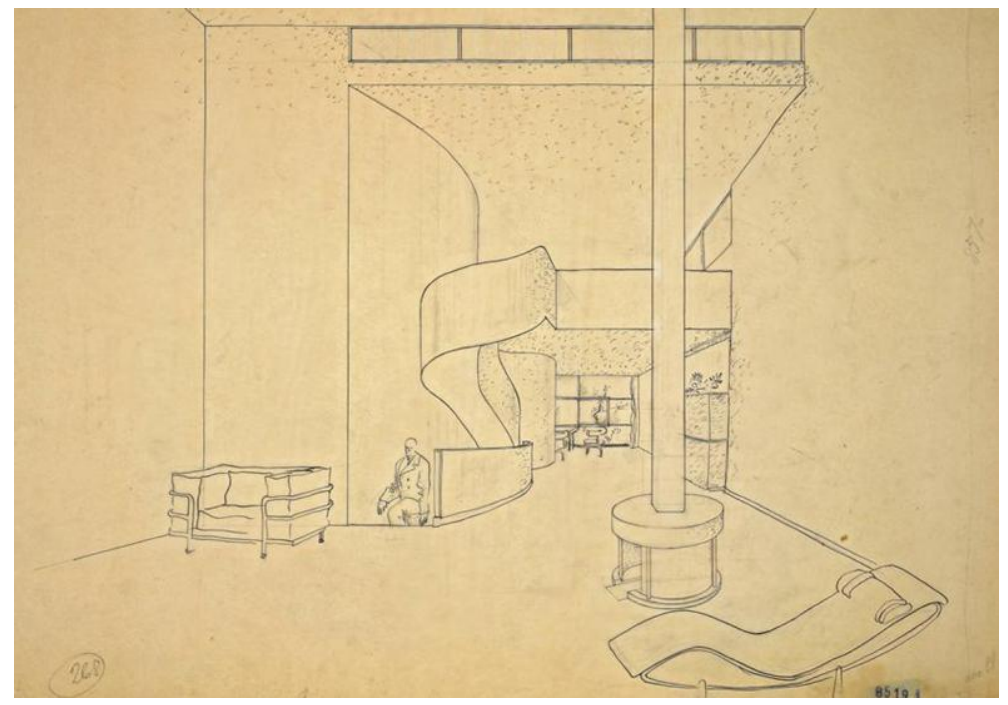

12. Maison Canneel (1929). Vue du salon et de la cage d'escalier.

A l'inverse, la richesse et la certitude des informations disponibles sur la Maison Guiette autorisent un mode d'expression beaucoup plus réaliste de l'hypothèse de restitution des couleurs et de l'aménagement mobilier aux alentours de 1940. Comme exposé précédemment dans cet article, la démarche de regroupement des informations au sein du modèle 3D tend vers un degré de certitude élevé. L'espace de la maison trouve alors son expression dans une simulation d'images numériques photo-réaliste, qui, une fois animées, permettent de profiter pleinement de la spatialité engendrée par l'hypothèse proposée (5).

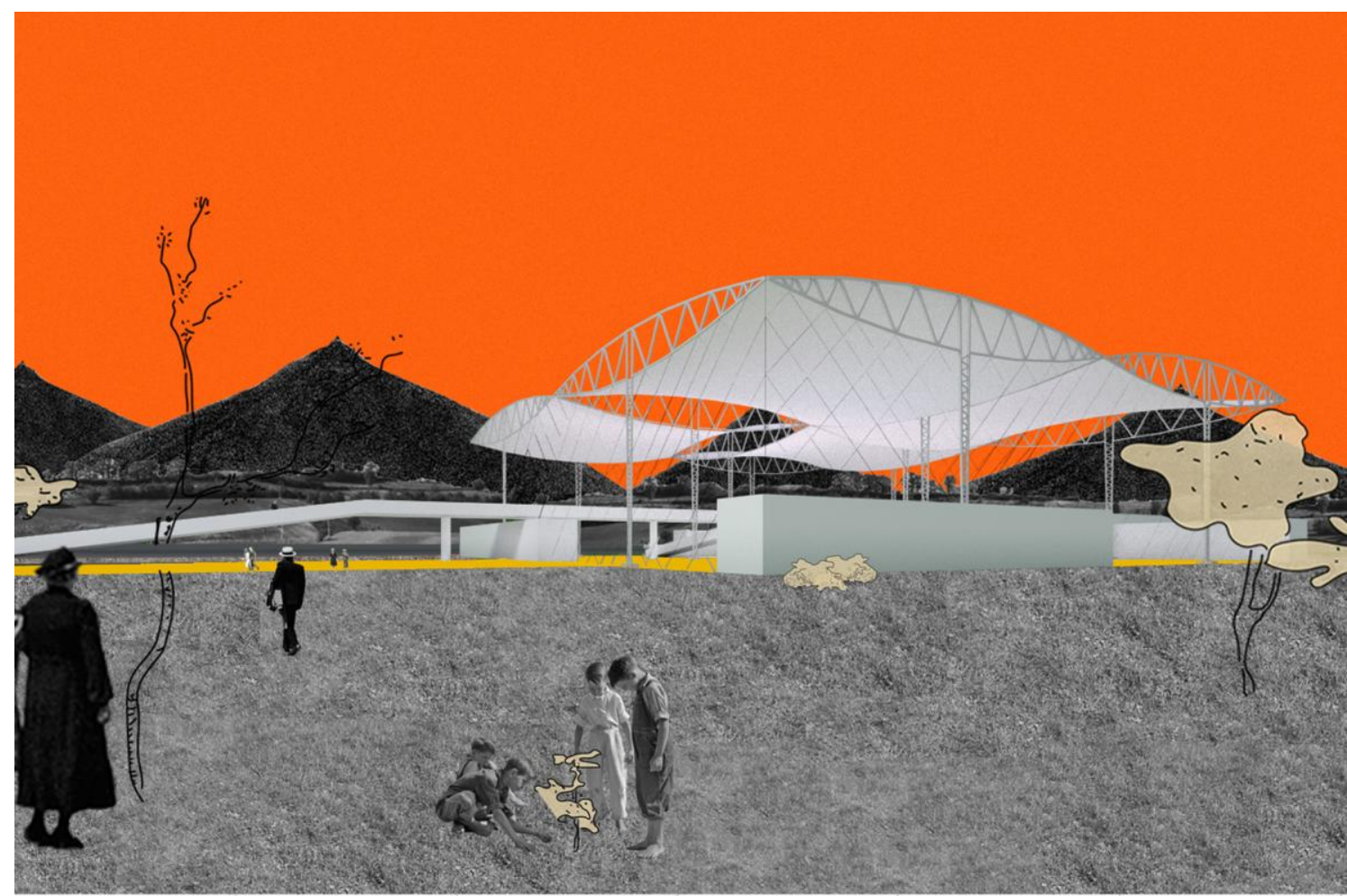

13. Pavillon de l'Exposition de l'eau (1939). Hypothèse de restitution d'une vue extérieure du projet.

Le peu d'informations disponibles ainsi que les diverses versions du projet Pavillon de l'Exposition de l'eau ont également déjà été abordées dans ce texte. Le modèle 3D regroupant les informations sur le projet, bien que très hypothétique, doit néanmoins trouver une manière d'être représenté en ne laissant aucune ambiguïté par rapport 
au niveau de connaissances que l'on a du projet. Un travail a donc été effectué sur une forme de représentation faisant la part belle au lyrisme et à l'aspect symbolique de la représentation architecturale. L'univers graphique coloré issus des dessins de l'architecte moderniste belge Renaat Braem (1910-2001) a servi de source d'inspiration pour suggérer les grands principes du projet et lui conférer une certaine matérialité, tout en restant résolument évasif et imprécis (13).

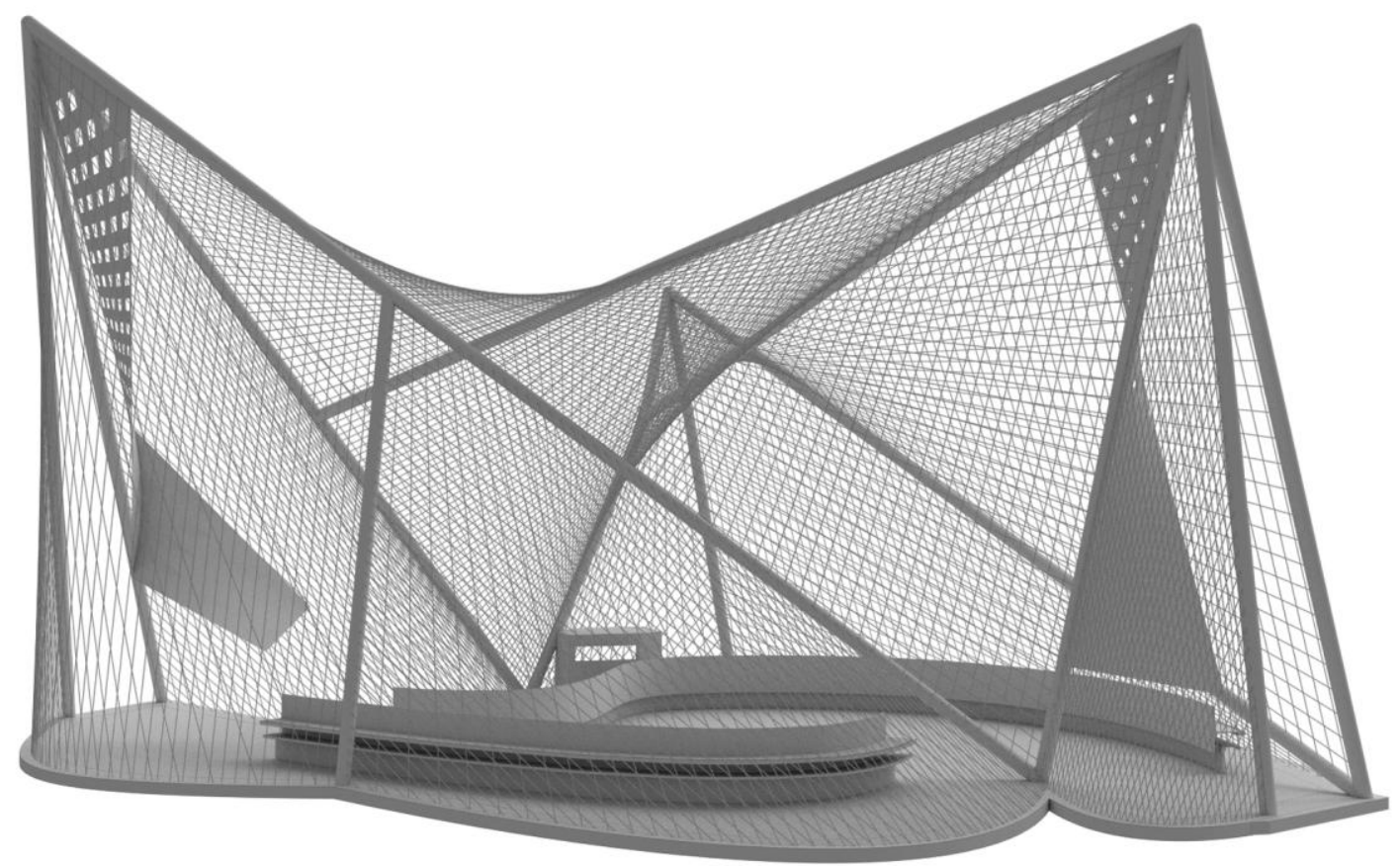

14. Pavillon Philips (1958). Hypothèse de restitution, développement didactique des principes structurels.

Le Pavillon Philips a déjà fait l'objet de plusieurs études - certaines très récentes ${ }^{28}$ - dont plusieurs travaux de modélisation 3D et de restitution par images de synthèse, notamment en ce qui concerne l'expérience sensorielle complète du Poème électronique qui était projeté à l'intérieur (Le projet VEP). Le travail des étudiants sur l'hypothèse de restitution $3 \mathrm{D}$ du pavillon Philips ne cherche donc pas à concurrencer des réalisations déjà très abouties, mais vise plutôt à proposer un document de synthèse, explicatif et didactique, depuis la commande du pavillon jusqu'à sa destruction, en passant par les divers épisodes de sa conception. En mêlant images d'archives et maquette 3D numérique, le court-métrage tend à décoder les différents composants de cet objet complexe, des poutres à la coque en béton avec les armatures extérieures, en utilisant avantageusement le modèle numérique pour rendre explicite la construction et le fonctionnement particulier de cet édifice. Le Poème électronique est aussi évoqué. Une reconstitution en est proposée, mais qui ne prétend nullement à l'exactitude précédemment atteinte. A la place, par une succession lancinante de points de vue grand angle depuis des endroits inaccessibles, le film propose une visite extatique et rêveuse du Poème. Afin de garder le propos clair et simple, le processus analytique du film utilise un mode d'expression qui renvoie à la maquette de concept : un objet dépourvu de détails reçoit une lumière neutre au milieu d'un contexte immaculé (14). Par contre, le court-métrage propose un

\footnotetext{
${ }^{28}$ Wever, Peter: Inside Le Corbusier's Philips Pavilion. A Multimedial Space at the 1958 Brussels World's Fair. Rotterdam : Nai010 Publishers, 2015.
} 
rendu plus réaliste de la peinture métallisée recouvrant l'extérieur de la coque en béton, l'extérieur du pavillon ayant été traité de manière plus simple dans les reconstitutions 3D existantes.

\section{Conclusion}

Comme nous l'avons exposé, notre laboratoire encourage une réflexion sur les moyens de représentation numériques dans un contexte analytique plutôt que dans un contexte de conception, pour la simple raison que l'analyse architecturale peut être considérée comme une forme de rétro-conception, mais vierge - dans une certaine mesure - d'idéologies architecturales. Il est dès lors possible de se focaliser uniquement sur l'objet à étudier, sur les intermédiaires graphiques qui permettent sa manipulation, de comprendre les codes intrinsèques de la représentation graphique, et d'ensuite imaginer comment transposer ces derniers dans le média numérique animé. Dans le cas de l'étude de l'oeuvre belge de Le Corbusier, la plus-value apportée par ce type de travaux est d'ordres divers. Dans le cas de projet non-réalisés et relativement peu documentés comme le Pavillon de l'Exposition de l'eau ou la Maison Canneel, les documents graphiques produits ont un statut hypothétique et exploratoire qui leur confère une certaine singularité : plutôt qu'une représentation exacte du projet, ils sont plutôt un regard ou une interprétation, qui relève plus d'un état de connaissances sur le projet dans un contexte particulier, que de l'énonciation illusoire d'une vérité absolue. Dans le cas du Pavillon Philips ou de la Maison Canneel, l'apport concerne également la mise en évidence explicite de questions compositionnelles ou constructives complexes. La contribution à la connaissance sur la Maison Guiette est plus ténue étant donné la documentation déjà abondante sur le bâtiment. Le travail cherche dès lors à explorer des questions de détail en exploitant la richesse des ouvrages et des archives existantes. Dans tous les cas, un des apports notables de ces restitutions par le modèle 3D et l'image numérique, c'est de proposer une expérience cinétique de la spatialité hypothétique du projet.

Pour produire le média que constitue un court-métrage analytique, il y a nécessité de produire de nombreux autres médias, de nombreux autres objets graphiques. Le film, une fois terminé, est bien plus qu'un fichier numérique animé diffusable. Il est surtout caractérisé par son processus de création qui contient bien plus de connaissances sur l'objet étudié que le document final. Ce processus est traçable car archivé et référencé. En outre, la modélisation 3D d'une architecture est indissociable d'une compréhension exhaustive de l'objet étudié. Dans un processus de modélisation, une décomposition de l'architecture est nécessairement suivie d'une recomposition, d'un encodage méthodique. Ainsi, au-delà de la géométrie, le modèle 3D devient une base de connaissances sémantiques, une nouvelle source d'étude sur l'objet qu'il représente. Si les méthodes d'élaboration passent successivement et sans apriori des méthodes traditionnelles aux médias numériques, le résultat final est lui résolument contemporain : il profite d'Internet et des réseaux sociaux ${ }^{29}$ pour diffuser le savoir architectural.

\section{Sources des images}

1, 2, 6, 7, 9, 11. AlICe (ULB), 2015. Tom Pariente, Jérémy Moreau.

3, 13. AlICe (ULB), 2015. Marine Serrette.

4. René Guiette.

5. AlICe (ULB), 2015. Quoc Anh Bach, Correntin Werrie.

8, 10, 12. Archives de la Fondation Le Corbusier, ADAGP.

14, 15. AlICe (ULB), 2015. Benoit Capel, Jonathan Van Saet, Geoffrey Minne.

\footnotetext{
${ }^{29}$ Les productions du laboratoires sont accessibles par le site http://www.alicelab.be/ et par le réseau d'échange de contenu video Vimeo : http://www.vimeo.com/alicelab/.
} 


\section{Bibliographie}

Baines, Georges: "Du «système Dom-Ino » au «type Citrohan » : la maison Guiette à Anvers ", En Massilia, 2011. pp. 50-81.

Baines, Georges; Spitaels, Els: Le Corbusier te Antwerpen: De Woning Guiette. Antwerpen : Hoger Architectuurinstituut van het rijk-stad Antwerpen, 1987.

Boesiger, Willy; Storonov, Oscar: Le Corbusier et Pierre Jeanneret. Oeuvre Complète. 1910 - 1929. Zürich: Editions Girsberger, 1943.

Boudon, Philippe; Pousin, Frédéric: Figures de la conception architecturale: manuel de figuration graphique. Paris: Editions Dunod, 1988.

Burniat, Patrick (Ed.): Le Corbusier et la Belgique (Les Rencontres de la Fondation Le Corbusier). Bruxelles: CFC-Editions, 1997.

Commers, Ronald; Lombaerde, Piet: Le Corbusier en de Linkeroever te Antwerpen. Antwerpen: Hoger Architectuurinstituut van het Rijk (H.A.I.R.), 1987.

De Heer, Jan: The Architectonic Colour: Polychromy in the Purist Architecture of Le Corbusier. Rotterdam: Nai010 Publishers, 2009.

De Kerckhove d'Ousselghem, Manoela; Goyens de Heusch, Serge: René Guiette. Antwerpen: Mercatorfonds, 1991.

Derycke, Denis; Lo Buglio, David: "Education in Architectural Analysis through Hybrid Graphical Means: A Setup for Critical Thinking". En Design \& Graphic Palimpsest. Atlanta GA: Southern Polytechnic State University, 2014. pp. 306-12.

Devos, Rika: Modern at Expo 58. Discussions on post-war architectural representation. Director: Mil De Kooning. Gent Universiteit, 2008.

Dumont d'Ayot, Catherine; Benton, Tim: Le pavillon de Le Corbusier pour Zurich : modèles et prototype d'un espace d'exposition idéal. Zurich: Lars Müller Publishers, 2013.

Evans, Robin: "Translations from Drawing to Building”. En Translations from Drawing to Building and Other Essays. London: Architectural Association Publications, 1997. pp. 153-193.

Jenkins, Eric J: Drawn to Design. Analysing Architecture Through Freehand Drawing. Basel: Birkhäuser, 2013.

Le Corbusier: Salubra, Claviers de couleur. Bâle, Editions Salubra, 1931.

Léglise, Michel: "Ordinateurs dans l'apprentissage de la conception : mental et instrumental". En Computerised Craftsmanship. Paris: eCAADe Conference Proceedings, 1998. pp. 138-145.

Lombardo, Vicenzo and others: "A Virtual-Reality Reconstruction of Poème Electronique Based on Philological Research". En Computer Music Journal. "A Virtual Poème”. Summer 2009, №2. Cambridge Ma: MIT Press, 2009. pp. 24-47.

Malliet, Anne: "Le Corbusier huis Guiette gerestaureerd”. En $M \& L$. November-december 1987, №6, 1987. pp. 49-60.

Mandoul, Thierry: “Une histoire dessinée”. En Entre raison et utopie. L'Histoire de l'Architecture d'Auguste Choisy. Wavre: Mardaga Editions, 2008. pp. 111-162.

Petit, Jean (Ed.): Le Poème Electronique - Le Corbusier. Paris : Editions de Minuit, 1958.

Picon, Antoine: “Architecture et mémoire numérique”. En Archigraphy. Paris: Infolio, 2007. pp. 55-63.

Prelorenzo, Claude (Ed.): La conservation de l'œuvre construite de Le Corbusier (Rencontres de la Fondation Le Corbusier). Paris : Fondation Le Corbusier, 1990. 
Prelorenzo, Claude: Le Corbusier et la couleur (Rencontres de la Fondation Le Corbusier). Paris: Fondation Le Corbusier, 1992.

Sakarovitch, Joël: Épures d'architecture: de la coupe des pierres à la géométrie descriptive XVIe-XIXe siècles. Basel: Birkhäuser, 1998.

Schneider, Bernhard: "Perspective Refers to the Viewer, Axonometry Refers to the Object". En Drawing as Medium of Abstraction. Daidalos - Berlin Architectural Journal, September 1981, $\mathrm{N}^{\circ} 1$. Berlin: Wilhem Krümpelmann, 1981. pp. 81-95.

Schraenen, Guy: Les Peupliers. Maison Guiette = Huis Guiette. Le Corbusier 1926. Brussel : Sint-Lukasarchief, 1987.

Sterken, Sven: Iannis Xenakis, ingénieur et architecte. Director: Bart Verschaefel. Gent Universiteit, 2004.

Treib, Marc: Space calculated in seconds. New Jersey : Princeton University Press, 1996.

Wever, Peter: Inside Le Corbusier's Philips Pavilion. A Multimedial Space at the 1958 Brussels World's Fair. Rotterdam: Nai010 Publishers, 2015. 